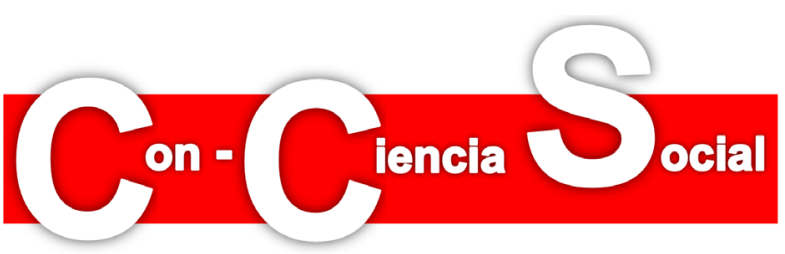

\title{
Comunicación y evaluación del conocimiento académico: tecnocracia y mercado
}

\section{Communication and evaluation of academic knowledge: tecnocracy and market}

\author{
M. Engracia Martín Valdunciel \\ Universidad de Zaragoza. Biblioteca y Fedicaria-Aragón \\ marien@unizar.es
}

Recibido en octubre de 2019

Aceptado en diciembre de 2019

DOI:10.7203/con-cienciasocial.3.16789

\section{RESUMEN}

La evaluación en general y la de sistemas educativos, en particular, es una práctica que se ha ido globalizando desde fines del siglo pasado. Institucionalmente se presenta como un mecanismo inevitable de modernización e internacionalización de la educación o de la investigación de acuerdo a estándares de ámbito europeo o mundial.

En este trabajo se mantiene la tesis de que la evaluación académica constituye un dispositivo para disciplinar instituciones y sujetos. Para abordar el tema contextualizaremos, en primer lugar, la expansión de la cultura de la auditoría porque creemos que la perspectiva histórica, económica y política enmarca el acontecimiento a escala global y le da sentido como dispositivo de poder. En segundo lugar, analizaremos el mecanismo a través de algunas instancias y discursos clave que lo sostienen. Por último, expondremos las limitaciones de algunos cuestionamientos al sistema evaluador.

Palabras clave: evaluación, Nueva Gestión Pública, conocimiento, neoliberalismo, Estado competidor, dispositivo

\begin{abstract}
Evaluation of education systems is a discourse practice that has spread throughout the world since the end of the last century. It is usually presented as a mechanism for modernising education or research according to European or world-wide standards. In this article it is sustained the thesis that academic evaluation is structured as a tool to discipline institutions and subjects. Firstly, the audit culture is contextualized because the historical, economic and political perspective clarifies the phenomenon on a global scale and gives it meaning as a mechanism of power. Secondly, the role played by some key instances and discourses are analyzed. Finally, the limitations to some questions about the evaluation system are presented.
\end{abstract}

Keywords: assessment, New Public Management, knowledge, neoliberalism, workfare state, device

\section{Referencia}

Martín Valdunciel, M. E. (2020). Comunicación y evaluación del conocimiento académico: tecnocracia y mercado. Con-Ciencia Social (segunda época), 3, 31-56. DOI:10.7203/concienciasocial.3.16789 


\section{EL MARCO}

\section{Cultura de la auditoría ${ }^{1}$ y NueVa Gestión PúBlica}

La conformación de un mercado global de la educación y de la investigación hay que enmarcarla, a nuestro entender, en un conjunto de fenómenos que tendrán una dimensión planetaria y que modificarán las estructuras económicas, políticas y sociales que caracterizaron el capitalismo embridado, como el extraordinario auge del capital transnacional, financiero y especulativo, potenciado por la tecnología electrónica, la reformulación del rol de los Estados, el crecimiento de las asimetrías entre la relación capital-trabajo o la sustitución de la actividad política por protocolos tecnocráticos de relación social.

En estos nuevos contextos sociales en que se generaliza la cultura de la auditoria los sujetos políticos se disuelven y devienen meros consumidores (stakeholders) que participan, de forma muy asimétrica, no ya en relaciones políticas sino en la gobernanza de la propia sociedad. No resulta extraño, por tanto, que muchos autores subrayen el déficit democrático en el funcionamiento de instituciones desde el último tercio del siglo pasado (Sassen, 2007; Jessop, 2008). Así, la gobernanza será la forma de gestión que adopta la universidad del tardocapitalismo en la que la cultura de la auditoría se normaliza.

El fenómeno ha sido escrutado desde enfoques propios de diferentes ciencias sociales. Desde la sociología política se ha analizado cómo los Estados asumen un rol relevante al acomodar territorial e institucionalmente la economía global en diferentes países mediante el fortalecimiento de regulaciones precisas o de procesos de privatización o mercantilización proyectando, al mismo tiempo, la lógica económica a todas las esferas de la vida. Es importante tener en cuenta este aspecto para no conceptualizar de forma errónea lo "público", pues de este tráfago de relaciones e influencias surgirá un nuevo tipo de autoridad estatal, un Estado competitivo, que no es ni totalmente pública ni totalmente privada² ${ }^{2}$.

\footnotetext{
${ }^{1}$ La cultura de la auditoría es una práctica discursiva deudora de la racionalidad eficientista. Surge para regular el medio financiero, pero se extenderá, desde el último tercio del siglo pasado, a toda la sociedad. Su lógica implica el incremento de la autoridad de agencias y expertos y la constante supervisión burocrática de instituciones o sujetos para medir sus outputs en función de su grado de adecuación a estándares (Shore, 2008; Craig, Amernic,Tourish, 2014).

2 En España, el Estado competitivo financia la universidad en función de "resultados", o aplicando categorías como "racionalización" o "sostenibilidad", sobre todo, desde el RD 14/2012, de forma que se estimula y promueve la financiación privada y se facilita a empresas o entidades financieras, como el Banco de Santander, la creación de "cátedras" que llevan su marca en un contexto regulatorio de gran opacidad.
} 
Los discursos y las prácticas de legitimación del sistema que emergen y se extienden desde el último tercio del siglo pasado se basan en conceptos como "calidad total" o "nueva gestión empresarial" que suponen, de facto, modos de dominación gestores. Categorías que van a tener una amplia resonancia y se van a materializar en el medio institucional público, especialmente en el ámbito educativo. Implican la puesta en marcha de toda una ortopedia que normaliza y reduce a mercancías realidades muy diversas: sistemas educativos, instituciones, campos de conocimiento, formatos de publicación, etc. ${ }^{3}$, para que entren a competir. La ficción tecnocrática y positivista, que se arroga neutralidad y objetividad científica, se apoya en la cuantificación y la comparación (Bruno, 2008).

Categorías como lex mercatoria, derecho corporativo global, privatización del derecho, etc., caracterizan la capacidad del poder económico, sobre todo el transnacional, a la hora de materializar una arquitectura jurídico-normativa y procedimental que blinda y legitima el capital de las corporaciones con el concurso de Estados, think tanks u organismos internacionales (FMI, OMC, Banco Mundial, Comisión Europea, etc.) frente a la devaluación y desprotección de los derechos sociales y humanos (Hernández Zubizarreta, Ramiro, 2015; Estévez Araujo, 2016). Así, los intereses económicos se ven protegidos por "derecho duro", mientras que las obligaciones se resuelven en auto-regulaciones, o "derecho blando" (es decir, no vinculante), como la tan extendida, también en el ámbito universitario, responsabilidad social corportativa.

En este contexto se va a desarrollar toda una constelación de prácticas discursivas relacionadas con auditorías, certificaciones, evaluaciones, sellos de calidad, etc., que llevan a cabo "agencias" a las que se ceden funciones que solían considerarse inherentes a la soberanía estatal (y, por tanto, con cierto nivel de regulación y supervisión). Se trata de un gran negocio, muy opaco, que escapa al control democrático, cuyo objetivo es articular una neo-burocracia al servicio de la homologación, medición y clasificación de sujetos o instituciones. Estos procesos, basados en el positivismo de los datos y la procedimentación tecnocrática, resultan clave para naturalizar la competitividad y la forma empresa. Como se ha avanzado,

\footnotetext{
${ }^{3}$ Es importante recordar el papel de agencias a escala europea orientadas a perfilar e implementar indicadores y estándares para utilizar en comparativas, clasificaciones, etc., como la European Network of Indicators Designers (ENID), que surge en 2007 de la mano de la Comisión Europea. Por otra parte, resulta paradigmático en los procesos evaluativos el reduccionismo del formato de publicación, el paper, que ha devenido en unidad de producción y cuantificación en los nuevos procesos neo-tayloristas del capitalismo académico.
} 
el New Public Management, o gobernanza, ha sido adoptada por las universidades en los cambios habidos en el escenario del capitalismo global e implica que la actividad que llevan a cabo sea supervisada por comités de expertos conforme a criterios ad hoc. Además de un mecanismo de legitimación de las dinámicas y de disciplinamiento colectivo e individual, estos procesos cumplen el rol de mantener una de las esencias del capitalismo, la ficción de la elección, al informar, o ser "transparentes", a los agentes económicos.

Desde la filosofía política se ha caracterizado el nuevo tipo de sociedades que se van generando en este marco como sociedades de control (Deleuze, 1999). En ellas se tiende a abandonar la coacción externa de forma que la violencia se administra de manera más difusa y sutil, por ejemplo, a través de marketing, modos de dominación gestores, construcción de consensos o proponiendo marcos de juego. El dispositivo de la evaluación, al presionar, indirectamente, para conseguir resultados forma parte de esta panoplia de técnicas de gobierno que devienen indispensables en la formación de un nuevo ethos académico individualista, competitivo y de autopromoción (Amigot Leache, Martínez Sordini, 2010; Gómez Sánchez, Jódar Rico, Bravo Sánchez, 2015). Como mantiene Ball (2012, p. 18), el mecanismo traspasa a los sujetos: "gets into our minds and our souls, into the ways in which we think about what we do, and into our social relations with others". Todas estas perspectivas, macro y micro, conforman un crisol múltiple en el que diversas fuerzas actúan a diferentes escalas en las que se replica la cultura de la auditoría.

\section{ESPACIO EUROPEO DE INVESTIGACIÓN Y EVALUACIÓN EN LA UNIVERSIDAD ESPAÑOLA}

A lo largo de las últimas décadas las políticas de la Unión Europea la han ido develando como un proyecto del capital en el que la gestión sustituye a la política. Como se ha avanzado, la gobernanza es una forma de producción jurídica que se presenta como alternativa a la democracia representativa. En sus presupuestos ideológicos conceptos como ciudadanía o bien común desaparecen. Probablemente, la gobernanza sea conocida en el ámbito académico, sobre todo en España, desde hace unas décadas. Menos, quizá, como medio de gestión intergubernamental para modelar lo que se denomina Espacio Europeo de Investigación o Espacio Europeo de Educación Superior.

Especialmente intensa, esta forma de regulación se ha desarrollado en el marco de la Unión Europea en el que los intereses corporativos se han canalizado a 
través de lobbies, entre otros la influyente ERT (European Round Table of Industrialists) con enorme peso en el Parlamento Europeo y acceso a la alta burocracia de Bruselas a través de comisiones de expertos, agencias o producción de informes de toda laya (Balanya et al., 2005; Kaupinen, 2014). De tal forma que los intereses de las transnacionales se han visto reflejados en regulaciones diversas de la Comisión Europea, tanto en aspectos organizativos (por ejemplo, en la promoción del Open Method of Coordination), como la incentivación del uso de mediciones y comparativas, el impulso del discurso de las "economías basadas en el conocimiento", la defensa de la innovación y la emprendeduría, el apoyo a partenariados público-privados o el fomento de las regulaciones sobre propiedad intelectual (Bruno, 2008). Son posiciones que sigue manteniendo en la actualidad (ERT, 2009) y que informan, en buena medida, la "Europa 2020, una estrategia para un crecimiento inteligente, sostenible e inclusivo" de la UE que, a pesar de la situación del planeta y de la sociedad, mantiene, de forma persistente, un concepto caro al capitalismo, el "crecimiento".

Es importante detenernos brevemente en el Método Abierto de Coordinación por su trascendencia como práctica replicada a diferentes escalas. Nos referimos a un instrumento que, sobre todo a partir de la Estrategia de Lisboa (2000), sirvió para generar no una política europea sobre educación o investigación sino un marco de gobernanza que implicaba, de facto, una suerte de gestión empresarial a la que deberían acomodar su actuación los diferentes Estados e instituciones de la UE. Este tipo de regulación suponía el uso de indicadores, comparativas y controles evaluativos, elementos que se pondrán en marcha en diferentes niveles en los sistemas educativos o de investigación dentro del régimen del New Public Management.

En definitiva, puede concluirse que estas fuerzas jurídico-económicas son las que estructuran el Espacio Europeo de Educación e Investigación basado en la competitividad/innovación en el marco de las "economías basadas en el conocimiento". En esa línea, "Horizonte 2020", el Programa Marco de Investigación e Innovación de la UE, contempla, en un contexto de "crecimiento" y de "una gobernanza más fuerte", "garantizar que las ideas innovadoras se puedan convertir en productos y servicios que generen crecimiento y empleo".

Dentro de cada país el rol del Estado es clave porque "interviene en la reestructuración de la investigación en las universidades para alinearlas más 
estrechamente con las necesidades de los negocios, estimulando la gestión y explotación de la propiedad intelectual mediante empresas de base tecnológica (spins-offs), licencias, participaciones, parques científicos y tecnológicos, parques industriales, etc." (Jessop, 2008, p. 159).

La innovación y la lógica contable y eficientista configuran el marco en que surge el Espacio Europeo de Investigación como pieza clave de la Europa de la competitividad. Ese conjunto de elementos dibuja un tipo de gubernamentalidad (neoliberal) que establece las reglas del juego para que, libremente, los agentes se (auto)regulen: países, gobiernos, instituciones académicas, personal investigador, etc. No se trataba de integrar jurídicamente diferentes países, orquestar la diversidad institucional o arbitrar dinámicas locales específicas en un espacio único, sino de propiciar una (auto) disciplina indefinida a través de la competitividad para que los países, las regiones, las universidades... pudieran medirse libremente entre sí y a escala global. En este marco, el dispositivo de la evaluación resulta una pieza angular como mecanismo de homologación, producción y sometimiento.

En el contexto de los países de la UE las instituciones universitarias se han ido adaptando al nuevo escenario asumiendo la gobernanza como modus operandi y como forma de ir dando soporte a la arquitectura de lo que se ha denominado capitalismo académico: extensión de la racionalidad eficiente, gestión de intangibles o proyección como "marca" para competir por financiación y clientela en el mercado educativo o de investigación. En este escenario juegan un rol esencial las agencias de calidad ${ }^{4}$ que, como instancias de expertise, materializan y legitiman la cultura de la auditoría.

En España el Plan Nacional de Evaluación de la Calidad de la Universidad partió del Acuerdo del Pleno del Consejo de Universidades de 1995 por el que se aprobó el Programa de Evaluación Institucional de la Calidad de las mismas formalizando la primera convocatoria en1996-1997. Es decir, la lógica contable comenzó a establecerse en paralelo al proceso de convergencia con Europa, tal como se recogía en el monográfico de Revista de Educación (1998).

\footnotetext{
${ }^{4}$ A partir de 1998 una recomendación del Consejo de Europa invitaba a los Estados miembros a poner en marcha "sistemas transparentes de evaluación de la calidad". La ENQUA (European Network for Quality Asssurance) nace en el 2000 para el ámbito europeo; la ANECA surge en España a partir de la LOU, 2001; también se crearán agencias en diferentes Comunidades Autónomas. The Quality Assurance Agency for Higher Education de G. Bretaña había nacido en 1997; I'Agence d'Evaluation de la Recherche et de l'Enseignement Supérieur aparece en Francia en 2006, etc.
} 
Fueron varios los instrumentos legales e institucionales que emergieron en esta época en nuestro país, como la Ley de Universidades, 2001, o La Ley de la Ciencia, 2011, que ya incluían conceptos como innovación, transferencia, competitividad, relaciones universidad-empresa o mecanismos, hipotéticamente, objetivos para asignar recursos 5 .

En lo relativo a las agencias que forman parte del sistema contable, inicialmente fue la ANEP (La Agencia Nacional de Evaluación y Prospectiva) la que en 1986 comenzó a implantar una cultura de la evaluación. Su experiencia fue recogida por la Comisión Nacional Evaluadora de la Actividad Investigadora (CNEAI, 1989) con el objetivo de valorar la producción científica del profesorado universitario y de los investigadores del Consejo Superior de Investigaciones Científicas (CSIC). Inicialmente, la CNEAI se encargaba de la evaluación de sexenios del profesorado para valorar la obtención de complementos económicos, status o recursos (RD1086/1989). Sin embargo, una decisión que inicialmente había sido optativa terminó por convertirse en preceptiva desde 2012 ya que el personal sin sexenios quedaba expuesto a diversas formas de penalización, estableciéndose así una clara jerarquización entre el personal académico. Entre 1989-1995 se llevaron a cabo convocatorias generales y a partir de 1996 aquellas se publicaron por áreas de conocimiento, áreas que se han ido perfilando y definiendo desde 2005. Las actividades del CNEAI fueron asumidas por la ANECA en 2014, en la actualidad un organismo autónomo dependiente del Ministerio de Educación, Cultura y Deporte (RD 15/2014), encargada de acreditar instituciones, enseñanzas e investigadores.

En el proceso de inserción en el marco global, se han ido incorporando las referencias y métricas anglosajonas en las convocatorias de evaluación de la investigación. Se aplica una evaluación cuantitativa que tiene en cuenta la fuente donde se publica la investigación, no el trabajo en sí mismo. Los referentes para todo el proceso son, de forma prioritaria, los indicadores y publicaciones contenidos en los Science Citation Index, Social Sciences Citation Index, Arts and Humanities Citation Index, o JCR, de la empresa Clarivate Analytics en la actualidad.

\footnotetext{
${ }^{5}$ La Bayh Dole Act, EE.UU. 1980, supuso un claro referente para otras latitudes pues consolidó la posibilidad de patentar conocimiento producido en universidades financiadas públicamente. Complementariamente, surgieron diferentes estructuras intermedias para canalizar la relación universidad-empresa, como las OTRI (Oficinas de Transferencia de Resultados de Investigación), parques y plataformas tecnológicas, red de fundaciones universidad-empresa, espacios de excelencia, etc. Se favoreció a las empresas con incentivos fiscales, patentes de explotación, creación de otras empresas, etc., para que invirtieran en departamentos universitarios de investigación. En nuestro país las OTRIs nacen en 1988.
} 


\section{EL DISPOSITIVO Y SUS DISCURSOS}

\section{DE LA REVISTA ACADÉMICA A LOS OLIGOPOLIOS}

El interés político, social o económico de los grupos de poder por el control del saber a lo largo de la historia no es una novedad. Como es conocido, en la modernidad capitalista occidental comenzó a gestarse una racionalidad específica, el monocultivo científico (Shiva, 2008), que, paralelamente a la colonización, estigmatizó formas de conocimiento diferentes y jerarquizó como grupos subalternos a sus productores (Shiva, 2008; Sousa Santos, 2010). Así, lo que en la actualidad se denomina producción científica mundial se identifica, en realidad, con la lógica de conocimiento generado en occidente que, tomando como patrón la ciencia natural, dominó las dinámicas de procesamiento y difusión del conocimiento desde la modernidad. Países europeos hasta el estallido de la II Guerra Mundial y desde entonces, también, los EE.UU. fueron los espacios hegemónicos de producción y difusión de dicho saber (Barsky, 2014).

La historia de la ciencia y la comunicación de la misma, las publicaciones científicas, se ha basado desde su nacimiento en el siglo XVII en el trabajo organizado de sociedades científicas (al margen de la academia, muy a menudo) y ha fundamentado su prestigio en la práctica de la crítica (revisión por pares) consiguiendo un amplio reconocimiento. Abordar los cambios que tuvieron lugar en los procesos de difusión de la ciencia en la segunda mitad del siglo $X X$, que modificaron las estructuras de comunicación anteriores, es indisociable de las transformaciones que venimos describiendo.

Por de pronto, el panorama académico se modificó produciéndose el incremento y especialización de centros universitarios, un aumento exponencial de profesorado y estudiantes, fuertes inversiones en investigación (dirigida al control del espacio aéreo e industria de guerra en los EE.UU. o la URSS), se multiplicaron las dotaciones de las bibliotecas academicas, etc. Todos ellos constituyeron factores propicios para el aumento de las publicaciones y de las revistas científicas que conocieron una amplia demanda.

La tónica de comunicación en las décadas de los 60- 80 mantuvo una doble vía: por una parte, crece el número de revistas académicas de gran prestigio, con una significativa financiación en las universidades públicas, como medio de difusión 
de la investigación ${ }^{6}$. Por otra, se irá produciendo un incremento destacado en el número de empresas privadas que se dedican a editar trabajos académicos ya que se va consolidando como un negocio rentable. En este sentido, el caso de R. Maxwell y la editorial Pergamon es paradigmático de todo este proceso.

El relevo del procesamiento y difusión de la ciencia (cada vez de forma más generalizada, en lengua inglesa) dará un salto cualitativo cuando pase a oligopolios editoriales internacionales (ingleses, holandeses, norteamericanos), en ocasiones con estrechas conexiones con el mundo de la comunicación y otras líneas de negocio (Reig García, 2014) en un constante movimiento de fusiones y cambios. El proceso corre paralelo a dinámicas de concentración de capital y a la extensión de la cultura de la auditoría y el credencialismo en instituciones académicas. La penetración de los grandes oligopolios en asociaciones profesionales científicas o académicas ha sido notable al establecer acuerdos para gestionar sus revistas; de forma que publicaciones académicas de cierta tradición y prestigio se van a supeditar, estratégicamente, a corporaciones (marcas) adoptando frecuentemente la lengua inglesa como medio de comunicación.

Se abría así un nuevo marco -denominado modo 2 en la clasificación de Gibbons et al (1997)- en el que "la ciencia sale al mercado". El modo 2 es híbrido, tanto por los agentes que intervienen en la investigación (académicos o no) como por la forma de financiación (se canaliza y prima la privada); el conocimiento tiene aquí una meta clara: resolver problemas concretos, busca aplicabilidad y ya no será valorado por la academia o los pares sino por procedimientos de certificación de calidad privados en los que tienen peso y credibilidad revistas administradas por oligopolios. Pero, sobre todo, en el modo 2 se desarrolla una investigación orientada, específicamente, a generar beneficios (Gibbons et al., 1997). No es difícil reconocer en este modelo, a grandes rasgos, la situación de la academia del siglo XXI: presencia y presión de empresas y entidades financieras sobre determinados departamentos, orientación de líneas de trabajo hacia una investigación productivista, privatización del saber mediante patentes, difuminado de intereses público/privados, etc.

\footnotetext{
${ }^{6}$ El desarrollo de la ciencia en este contexto correspondería, siguiendo la clasificación de Gibbons et al. (1997), al tipo 1. El modo 1 identificaba un saber "tradicional" y abierto asociado a la universidad, enmarcado en disciplinas de conocimiento, que no disociaba de forma clara docencia-investigación, que no buscaba directamente rendimientos económicos y cuyos filtros de control se encontraban en los pares.
} 
Según se ha señalado, la confusión entre espacio privado-espacio público es un hecho que se acentúa desde la globalización a diferentes niveles, supraestatales, dentro de los países o en las instituciones académicas. Los intereses de grandes oligopolios de comunicación científica, los de los Estados emprendedores y sus agencias y los de las universidades parecen coincidir ya que aquellos han conseguido un fuerte protagonismo y credibilidad a la hora de difundir la producción del personal académico (Buranyi, 2017; Fyfe et al., 2017). Se trata de un gran negocio con repercusiones importantes que viene siendo fuertemente cuestionado, como puntualiza Barsky 2014):

La notable concentración editorial de quienes las editan [las revistas] ha devenido en un gigantesco negocio que además de afectar enormes intereses económicos, sacude las organizaciones académicas, genera patrones de subordinación a los procesos de evaluación y afecta los intereses de los académicos, sus márgenes de creatividad, así como esquematiza y deforma los procesos de evaluación apoyados en indicadores como el impacto de las publicaciones indexadas, cuya legitimidad ha sido crecientemente puesta en duda. (p. 97)

En la actualidad, se estima que existen entre 5.000 y 10.000 editores de revistas en todo el mundo. Sin embargo, unas pocas empresas concentran la publicación de más de 2.000 revistas cada una. Como ocurre en otros ámbitos, el libre mercado ha producido concentración y formación de oligopolios: en 2015 los conglomerados empresariales Reed-Elsevier (RELX), Taylor \& Francis, WileyBlackwell, Springer y Sage —las big-five_ gestionaban más del 50\% del total de las publicaciones científicas del mundo, las que cuentan a efectos credencialistas. Se trata de una industria que obtiene beneficios que superan el $30 \%$ y que, al haber adquirido una posición muy fuerte, tiene capacidad para fijar condiciones frente a los compradores, instituciones académicas y de investigación, fundamentalmente. Una situación que supone un triple pago público, como apunta Buranyi (2017, parr. 5): "el Estado financia la mayor parte de la investigación, paga los salarios de la mayoría de los que revisan la calidad de la investigación, y luego compra la mayor parte del producto publicado".

La eficacia del dispositivo depende, en buena medida, de ese rol que juega el personal académico e investigador, ahormado por una intensa arquitectura 
managerial, aún cuando bajo este término, sobre todo en el marco de la universidadempresa, se cobijan diferentes figuras con status e intereses muy diferentes.

\section{MÉTRICAS, ÍNDICES Y AGENCIAS}

Una de las preguntas que parece pertinente formularse, a tenor de los profundos cambios comentados anteriormente, es por qué en un determinado momento los procesos de comunicación científica implicaron, también, una valoración del currículum de investigadores e investigadoras. Paralelamente a las mutaciones en el modelo de publicación, se dieron otras relacionadas con los intentos por cuantificar la ciencia y con la globalización de las políticas educativas.

Las bases de datos que, hipotéticamente, indexan las mejores revistas y que integran lo que se conoce como Web de la Ciencia que, junto con la métrica del factor de impacto, constituyen el referente para valorar el trabajo de los investigadores de, prácticamente, todo el mundo desde hace unas décadas, surgieron en un contexto específico, los EE.UU. de la Guerra Fría; un fenómeno que es conveniente conocer, aunque sea someramente, para calibrar el alcance del dispositivo de la evaluación.

Como se ha indicado, el control sobre el saber por parte de instituciones educativas y culturales modernas no es una novedad y corrió paralelo al desarrollo y evolución de la disciplina de la Biblioteconomía, la Cienciometría, o la Sociología del conocimiento. Desde Europa, por ejemplo, investigadores como P. Otlet y H. La Fontaine, se habían hecho eco de ese interés al crear en 1895 el Instituto Internacional de Bibliografía de Bruselas en el reinado de Leopoldo II.

En el siglo XX, autores como J.D. Bernal o R. Merton prestaron atención a la posibilidad de definir indicadores fiables para calibrar el desarrollo exponencial de la ciencia, describieron el "efecto Mateo" o comenzaron a asociar el reconocimiento por parte de los pares y el número de citas recibidas por los investigadores con la calidad de su trabajo; una relación (reconocimiento = calidad científica) que será base conceptual de posteriores estudios sobre citación en revistas científicas.

Con la crisis del periodo de entreguerras decrecieron los presupuestos para la financiación o suscripción de revistas especializadas en los países desarrollados y se agudizó la presión por conocer y valorar la producción nacional de conocimientos. Desde la Bibliometría se hicieron diferentes aportaciones sobre técnicas de control documental. Así, las tesis del químico y documentalista S.C. Bradford mantenían 
que la mayoría de artículos de una especialidad concentran su difusión en unas pocas revistas. Identificar ese núcleo de publicaciones relevante podría servir de guía a la hora de priorizar financiación o suscripciones en las bibliotecas académicas. En los años 60, de Solla Price, considerado padre de la Cienciometría, fue uno de los precursores de los análisis cuantitativos de las citas en la investigación estableciendo una correlación entre aquellas y su solidez científica al tiempo que se interesó por definir núcleos de revistas de una disciplina.

En el marco de la Guerra Fría, el lanzamiento del Sputnik (1957) constituyó un acicate no sólo para repensar el sistema educativo norteamericano sino también para coordinar la producción y el control de la actividad científica de cara a competir por el control del espacio aéreo. En este contexto, se formó un grupo de estudio, con participación de empresas, Estado y expertos, que propuso el uso de índices de citas para comparar, jerarquizar o priorizar publicaciones.

Pero no fue un proyecto público aunque tuvo apoyo gubernamental. E. Gardfield, documentalista e investigador, propuso un sistema ${ }^{7}$ de selección de revistas y el uso de métricas, como el índice de impacto ${ }^{8}$, materializado y canalizado en los años 60 a través de su empresa, Institute for Scientific Information (ISI), que editó índices de citas relativos a revistas procedentes de diferentes áreas de conocimiento $^{9}$. Su pretensión, inspirada en las tesis de Bradford, era que quedaran identificadas las mejores. El apoyo de científicos relevantes, como de Solla Price, Merton o el nobel Lederberg, promocionó el proyecto de Garfield ante la comunidad científica, inicialmente muy reticente a sus propuestas, o ante diferentes instancias de poder, como la National Science Foundation, apremiada por racionalizar la inversión en investigación. ¿Cómo se dilucidó el núcleo inicial, esa élite de revistas, cuando en esa época se publicaban decenas de miles especializadas en el mundo y

\footnotetext{
7 En las propuestas de Gardfield, jugaron un papel relevante algunos factores, entre ellos, el conocimiento de índices de jurispridencia, los populares Shepard's Citations, un sistema por el que los abogados norteamericanos podían disponer de forma ágil de toda la jurisprudencia del país.

8 El factor de impacto, o índice de impacto, es un valor numérico que se establece para una publicación periódica en función del número de citas que reciben los artículos que contiene durante un período de tiempo determinado. Las métricas permiten comparar y clasificar las publicaciones. El Journal Citation Reports (JCR) es uno de los índices más utilizados en la evaluación académica junto con el SJR (Scimago Journal Country Rank). Se han desarrollado otros, como el h index, propuesto por Hirsch en 2005. Con la profusión de redes sociales y académicas se vienen proponiendo las altmetrics (métricas alternativas) basadas en el uso, consulta, comentario... de artículos en las redes, blogs, programas bibliográficos, etc. Así mismo, hay tendencias que confían en algoritmos matemáticos, como Google Scholar, para evaluar la investigación...

${ }^{9}$ En 1960 Gardfield lanza el Science Citation Index. Más tarde, en 1965, el Social Science Citation Index y en 1975 el Arts and Humanities Citation Index y el Journal Citation Reports (JCR). En 1992 Gardfield vendió sus índices a la empresa Thomson, más tarde Thomson Reuter. En la actualidad, las antiguas bases de datos del ISI pertenecen al consorcio Clarivate Analytics.
} 
en diferentes idiomas?

El sistema, ligado a editoriales poderosas, áreas de conocimiento tecnocientífico y al uso de la lengua inglesa, no dejó de ser arbitrario. Inicialmente, tanto el proceso de selección de revistas como el cálculo del índice de impacto, pretendían proporcionar información estadística de apoyo a las instituciones y las bibliotecas para conocer, financiar o suscribir las publicaciones más importantes o utilizadas. Sin embargo, la compleja red informativa y de intereses (las relaciones con investigadores y editoriales importantes o el negocio de la evaluación) se fue asentando y se llegó a identificar el uso / cita = bondad. Por extensión, la misma idea se extrapoló a los investigadores en función de la fuente en la que publicaban.

Como sostienen algunos autores, entre ellos Barsky (2014, p. 264), Gardfield "construyó un sistema parcial, limitado, deformado y luego la comunidad científica lo sacralizó". Una vez aceptado el pragmatismo de los datos y los productos de su empresa por ciertas élites, las limitaciones iniciales de la selección, entre ellas la escasez, se tornarían, como ocurre en la actualidad, en distinción ventajosa para las que están dentro con respecto a las ausentes. La utilidad de la emergente Cienciometría ${ }^{10}$ vendría más tarde a sancionar una realidad cerrada. Este sistema, que siguió creciendo y modificándose (Emerging Sources Citation Index), se extendió al resto del mundo a partir del último tercio del siglo pasado. A dicha Web, se añadieron, desde 2004, las bases de datos y métricas de otro oligopolio, Reed Elsevier (REXL): en la actualidad constituyen los referentes mundiales para medir, comparar y clasificar revistas y, por extensión, investigadores. El sistema ha tenido el mérito de constituir un verdadero régimen de verdad en la academia de forma que ha sido ampliamente consensuado en la globalización neoliberal. Se han venido manifestando discrepancias de matiz, pero difícilmente se pone en tela de juicio el propio régimen meritocrático como dispositivo de control.

\section{EL DISCURSO DE LA INNOVACIÓN}

Especial interés, por su trascendencia en las políticas de promoción o comunicación del conocimiento científico-académico dentro de la UE, tiene el

\footnotetext{
${ }^{10}$ El nacimiento de la revista Scientometrics (1979) suele tomarse como referente del surgimiento de la "ciencia de la ciencia". En su visión primarán los análisis cuantitativos en un contexto en el que comienza a experimentarse con la computación. Como acertadamente apuntan Callon, Courtial, Penan (1995, p. 11): "La cienciometría se inscribe en el momento de su nacimiento en una visión muy positivista de la actividad de investigación... en cierta forma se trata de cientifismo al cuadrado".
} 
discurso de la innovación, presente en el "Programa Horizonte 2020" o incorporado en nuestro país por la Ley 14/2011 de Ciencia, Tecnología e Innovación. En cierta forma, como apunta Sádaba (2008), ha reactualizado, implícitamente y en versión tecnocrática, el ideal de progreso tan caro a la modernidad. El discurso de la innovación se ha convertido en un a priori cargado de positividad base para legitimar los procesos de protección/cercado de conocimiento a diferentes niveles (Shiva, 2003; Khor, 2003). En instituciones académicas se materializa a través de plataformas y regulaciones que entrelazan intereses de empresas, Estados, universidades e investigadores ¿En qué consiste innovar? Desde la Comisión Europa la innovación es entendida como la capacidad de transformar conocimiento en riqueza constituyendo aquella el núcleo del espíritu empresarial.

Así, se produjo un dislocamiento de cierta trascendencia: la invención, la producción de conocimiento como "mundo abierto", se diferenciaba de la explotación comercial, espacio de competición económica. Desde el último cuarto del siglo XX, con nuevas regulaciones sobre propiedad intelectual y la entrada del capital financiero en todo el proceso de innovación (en campos como biotecnología y software) las diferencias se difuminan (Coriat, Orsi, 2005). Aunque es claro que nunca habían sido campos inconexos, se refuerza una alianza sin mediaciones. Por lo que se refiere a la universidad, al estatuto de la ciencia o los investigadores, el escenario descrito implicaba vías hacia un nuevo modelo, el citado modo 2 (Gibbons et al., 1997).

En este escenario, prevalece el valor de cambio del conocimiento y su sanción se supedita al cumplimiento de estándares de calidad regulados por agencias con capacidad para acreditar o certificar dentro del marco de la lex mercatoria. Los límites de la investigación, en última instancia, serán así marcados por la competitividad y los nichos de negocio que consideren pertinente las instancias de financiación. No hay que olvidar, por otra parte, la capacidad de provecho del saber "no rentable", pero con capacidad para legitimar el capitalismo (sobre todo en el campo de las ciencias sociales y humanidades).

Las asunciones antropológicas o jurídicas del discurso de la innovación proceden del pragmatismo liberal convenientemente puesto al día en el tardocapitalismo. Los sobreentendidos del discurso obvian la sociología y la historia de los procesos de acumulación colectiva de saberes: descontextualizan y reifican el conocimiento material o simbólico, al desligarlo de las sociedades o los países que 
lo producen, para que entre en el circuito de producción de valor. En la base conceptual del discurso de la innovación se encuentra un sujeto soberano que es capaz de inventar ex nihilo para contribuir al progreso social. Desde el lusnaturalismo liberal el producto de tal actividad se sancionará como propiedad natural derivada del trabajo individual, a la que corresponden beneficios asociados (patentes, citas, reconocimientos, etc.).

De la axiología liberal al eficientismo tecnicista contemporáneo, la innovación se instrumentaliza en la actualidad como incentivo para estimular la creación, base del crecimiento económico y la competitividad global. Los propulsores de la idea parecen indicar que sin estímulos económicos no hay generación de riqueza, como si la naturaleza misma o la historia de sociedades, instituciones o países no hubieran dado muestras de supervivencia y creaciones colectivas basadas en el hecho de compartir recursos y conocimientos no enajenables (Shiva, 2003; Khor, 2003). La visión dominante propone una concepción humana perezosa y egoista, el homo oeconomicus neoliberal, que no actúa si no es con la zanahoria de incentivos materiales obviando otros intereses morales $u$ otros acicates para la acción. La retórica de la innovación eleva, por tanto, a categoría antropológica el pragmatismo, el utilitarismo o la racionalidad económica.

El mantra es que la innovación y el empresariado se conforman como agentes de cambio mientras que las instituciones y la sociedad deben adaptarse, simplemente, a las dinámicas que generen. El discurso redunda en la idea, canalizada antes a través del "desarrolismo" de la segunda mitad del siglo XX, según la cual cualquier cambio de modelo social debe dejar hacer a la técnica, los expertos o los emprendedores. En resumen, esta ideología no hace sino apuntalar las estructuras de poder que conforman la "triple hélice" —Estado competitivo, grandes corporaciones del capitalismo financiero global y universidad-empresa- para capturar, sobre todo, el conocimiento tecno-científico motor de un capitalismo desbocado que ya ha evidenciado peligrosamente sus límites.

\section{EL DISCURSO DE LA INTERNACIONALIZACIÓN DE LA CIENCIA}

El concepto internacionalización de la ciencia para referirse a un aspecto de la globalización actual se encuentra omni-presente en las políticas académicas y de investigación de todo el mundo. El concepto "internacional", según el DRAE (Diccionario de la Real Academia), significa: "perteneciente o relativo a dos o más 
naciones". Sin embargo, la definición no da cuenta de la asimetría existente en las relaciones de diferente tipo entre países. Como es sabido, la mayor parte del planeta se encontraba, desde la modernidad y hasta hace apenas unas décadas, bajo el dominio de algunas naciones europeas y, más tarde, de los EE.UU., hecho que deja patente esa relación desigual.

Así mismo, es sabido que la dominación tiene diferentes dimensiones. Han podido desaparecer las más groseras mientras se mantienen aquellas que se imbrican en culturas e imaginarios. Investigadores como Said (1996) han demostrado la eficacia de la producción simbólica de las potencias europeas para crear identidades y formas de naturalización de relaciones jerárquicas entre sociedades y países que aún persisten. Quijano (1999) evidencia que, si bien ha habido procesos (políticos) de descolonización, la colonialidad del poder se mantiene a través de la imposición/asunción del imaginario eurocentrista a lo largo de los siglos, sobre todo en los grupos sociales dominantes.

Por otra parte, el idioma de comunicación del saber no es un aspecto secundario. Hasta mitad del siglo XX las lenguas que vehiculaban el conocimiento moderno fueron el francés, inglés y alemán, principalmente, y se correspondían con la hegemonía política y económica de los países de referencia. Desde el fin de la II Guerra Mundial, el uso de la lengua inglesa se ha ido imponiendo. En marcos de gestión tecnócrata, suele presentarse la generalización del inglés como mero instrumento aséptico de intercomunicación científica mundial obviando su relación con las estructuras de poder y con el hecho de que una lengua canaliza ideas, valores y cosmovisiones del país de procedencia (Phillipson, 2000).

Desde el final de la II Guerra Mundial, el imperialismo de los EE.UU. ha sido capaz de americanizar el mundo desde diferentes frentes: fundamentalmente, a través de los mass media han difundido de forma eficaz el American way of life. Sería muy reduccionista pensar que el fenómeno se circunscribe a la esfera de la cultura de masas, sobre todo cuando el medio académico va asociándose cada vez más al de los grandes medios de comunicación. El imperialismo cultural afecta también a los campos de conocimiento a través de la producción de categorías, prácticas y conceptos asumidos por académicos e investigadores como mecanismo de legitimación y de reconocimiento, toda vez que los EE.UU. constituyen el referente de comportamiento en diferentes esferas, entre ellas el medio académicocientífico. 
Esta capacidad de influencia se hace evidente a la hora de proponer globalmente temas o problemas objetos de estudio y debate. Por lo demás, es conocido que detrás de muchos proyectos se encuentran potentes fundaciones privadas (Rockefeller, Ford...) think tanks, o empresas que orientan y sufragan líneas de investigación en función de sus intereses. En realidad, podría hablarse de "americanización" toda vez que el imperio intenta "universalizar" su visión del mundo (Bourdieu, Wacquant, 2012), al internacionalizar la edición universitaria, al desaparecer la diferencia entre editoriales universitarias / comerciales o al extrapolar a otros países los mecanismos, instrumentos o índices específicos que se han mencionado.

La misma idea de desigualdad se constata si contextualizamos la internacionalización de la ciencia en la geografía estratégica de la globalización (Gómez Morales, 2017). Más que de un diálogo entre iguales se trata de una competencia desequilibrada entre el Norte y el Sur global ${ }^{11}$ que recuerda la desproporción entre David y Goliat (Beigel, 2008). Como afirma Valdecantos (2009):

como quiera que dentro de la burocracia de los rankings y de los títulos de prestigio, el predominio de las universidades estadounidenses no admite competencia, la internacionalización significa en la práctica la integración provinciana y colonial en los paradigmas que tengan vigencia en los Estados Unidos. (p. 10)

En este sentido, la evaluación, elemento clave en la internacionalización, opera como un mecanismo más de dominación geopolítica enmarcado en el discurso del "mercado global"12 (Mignolo, 2003). La mundialización de la ciencia tiene como base un saber-poder que detenta el mundo occidental sustentado en verdades que se autodemominan científicas, avanzadas, superiores, globales y productivas (Sousa Santos, 2010). Este hecho implica la producción de la no existencia epistemicidio- de realidades específicas, colectividades, sujetos y discursos, que esa perspectiva sitúa en países o colectivos subalternos. El saber-poder dominante

${ }^{11}$ El concepto Norte-Sur global procede de B. de Sousa Santos: se refiere metafóricamente a comunidades en posición subalterna, independientemente de su localización geográfica.

12 Según Mignolo (2003), las múltiples dimensiones de la colonización occidental se han apoyado en diferentes discursos: la misión cristianizadora (protagonizada por la Iglesia y el capitalismo mercantil en nombre de la fe), la misión civilizadora (llevada a cabo por Estados-nación y por el capitalismo industrial bajo lemas como razón o progreso) o la misión desarrollista, liderada por el imperialismo de los EE.UU. y corporaciones internacionales. En la actualidad, la misión globalizadora es indisociable de organismos como la Organización Mundial de Comercio y el capitalismo financiero. 
aliena el conocimiento, las prácticas y discursos locales tachándolos de inferiores, atrasados o inadecuados. Lo cual no es óbice para, a través de la aplicación y coerción de la lex mercatoria (OMC y ADPIC), patentar y privatizar cultura y conocimientos relacionados de forma amplia con la vida.

En realidad, subyace a la internacionalización de la ciencia una estrecha conexión entre conocimiento/capital global que beneficia a grandes corporaciones pues, en realidad: "no tiene relación con las necesidades humanas, el $90 \%$ de esa producción de conocimiento podría detenerse sin riesgo de privaciones para los seres humanos" (Shiva, 2008, p. 77-78). Más aún, buena parte de la tecnociencia se convierte, de facto, en un "proyecto de muerte" —muy evidente en la "investigación de defensa", en episodios recientes como los de Bhopal, Chernobyl, etc., o en la potencia destructora de corporaciones farmacéuticas o agroquímicas, como Sandoz o Monsanto.

En definitiva, con ese sistema de intercomunicación y valoración del saber se promueven estrategias que dañan la autonomía e impulsan la subordinación de intereses, lenguas y medios de comunicación del Sur global: "todo ese esfuerzo de los científicos locales por parecerse a los científicos desarrollados resulta, en su mayor parte, irrelevante en términos internacionales (pocas o ninguna cita) y no pertinente en términos locales o nacionales" (Gómez Morales, 2017, p. 282). Por consiguiente, hay unas claras jerarquías epistemológicas y políticas que el ideario de la internacionalización obvia promoviendo un diálogo ficticio y una falsa objetividad evaluativa que deviene medio para naturalizar y mantener las diversas desigualdades.

\section{Cuestionamientos, RESISTENCIAS, SOLUCIONISMO TECNOLÓGICO}

Las críticas y objeciones al sistema y a su uso/funcionamiento no han dejado de existir desde el inicio: desde el cuestionamiento de la selección de las publicaciones referentes a problematizar la idea de que una cita sea considerada sinónimo de bondad — cayendo en la tautología un paper se cita porque es bueno, luego es bueno porque se cita-, pues, básicamente, la mención mide el consumo; de ahí no puede colegirse, necesariamente, la bondad del contenido de un texto. Además, se ha cuestionado la base de operatividad del índice de impacto: los responsables de los índices estarían haciendo un uso reduccionista de la cita bibliométrica valorando básicamente su dimensión formal y obviando su sentido 
científico y cognitivo (Bermejo Barrera, 2006).

Los investigadores de áreas como las Ciencias Sociales o las Humanidades, principalmente del Sur global, no han dejado de expresar su disconformidad con la lógica de las métricas que toman como referente los contenidos de la Web de la Ciencia y de su aplicación en el marco curricular, defendiendo en ocasiones evaluaciones cualitativas. Principalmente, por su situación de manifiesta desventaja con respecto a colegas de áreas científico-técnicas, disciplinas con más representación en las revistas mainstream o por la primacía del paper sobre otros formatos de publicación (Reig García, 2014; Herrán, 2012).

También se ha puesto de manifiesto que el sistema de evaluación da preeminencia al conocimiento empírico sobre análisis teóricos o críticos, prestando una mayor atención a perspectivas epistemológicas positivistas y estrategias metodológicas cuantitativas. Es un sistema que prioriza la acumulación, gestión y consumo de información, lo que beneficia a los oligopolios citados o entidades académicas meritocráticas, en detrimento de la capacidad reflexiva o la experiencia vital de sujetos y colectivos. En este contexto, se ha problematizado el rol de las Ciencias Sociales que puedan cuestionar el statu quo ya que sólo se considerarán "útiles" en la medida en que se supeditan a la legitimación de la producción (Bruno, 2008).

Así mismo, hay literatura abundante que denuncia "malas prácticas" o "conductas inapropiadas" relacionadas con el conjunto del sistema actual de evaluación: desde el "todo vale" a la hora de situar estratégicamente revistas en puestos competitivos, al abultamiento de citas, o "redes de citas", coerción de evaluadores y editores de revistas sobre los autores, connivencias entre redes de investigadores, etc., etc. Una cuestión a plantear aquí sería hasta qué punto el propio sistema, basado en la competitividad a ultranza, no forma parte del problema.

Frente al abuso del mainstream como referente base, surgieron diferentes proyectos en países latinoamericanos, o en España, algo más tarde, que no cuestionaban de forma radical el sistema, pero sí las fuentes o los indicadores, y cuyo objetivo se centraba en construir índices y clasificaciones más acordes con la realidad de los países de referencia ${ }^{13}$. Sin embargo, en España desde 2009-2010

${ }^{13}$ Desde 1994 existe un Sistema Regional de Información en Línea para revistas (Latindex) que prioriza la visibilidad de publicaciones en lengua española. O la Red de Revistas Científicas de América Latina y el Caribe, España y Portugal (Redalyc, 2003). En la misma línea, podría citarse SciELO (Scientific Electronic Library Online). En nuestro país, ya a principios del presente siglo comienzan a consolidarse proyectos en ámbitos como las Ciencias Sociales y las Humanidades, 
han dejado de financiarse y de actualizarse, lo que significa, de facto, ceder espacio a los referentes hegemónicos en los procesos de evaluación (Giménez Toledo, 2014).

Recientemente, han surgido cuestionamientos colectivos, como la Declaración de San Francisco (2012), que se posiciona claramente frente al uso de métricas basadas en el impacto de las revistas y postulan la necesidad de valorar un trabajo de investigación en sí y por sus resultados, no en función de la fuente en la que se dé a conocer. En 2012 también la campaña The cost of knowledge evidenciaba el descontento de parte de la comunidad académica por el negocio de Elsevier e invitaba a boicotear el oligopolio evitando publicar, editar o revisar trabajos académicos para el mismo.

Desde 2016 varias instituciones académicas alemanas cancelaron sus suscripciones con Elsevier y otras grandes editoriales en protesta por sus contratos leoninos; lo propio ocurrió con bibliotecas universitarias finlandesas y de los Países Bajos. En España no se conoce nada similar. A través de la FECYT se sigue manteniendo el pago millonario a Clarivate y Elsevier que, además, canaliza e incentiva su uso a través de "módulos de formación" a comunidades investigadoras o bibliotecarias. Por otra parte, si bien desde Rebiun (Red de bibliotecas universitarias) se defiende el libre acceso a la investigación, las bibliotecas académicas se hacen eco del discurso dominante dando cabida, por ejemplo, a "talleres de autores" en los que se promociona la lógica eficientista, el modus operandi o, directamente, los productos de Elsevier.

El Manifiesto de Leiden (2015), respaldado por "bibliómetras", plantea mejorar la metodología ya que, mantienen, los sistemas de evaluación no utilizan las métricas de forma correcta. En España, colectivos como Indocentia o Unidigna, convocan a académicos e investigadores a reflexionar sobre el contexto actual y cuestionar la cultura del impacto al tiempo que defienden una investigación al servicio del conjunto social. En la misma línea se encuentra el proyecto Amelica que surgío en 2018 a partir de la colaboración con Redalyc, el Consejo Latinoamericano de Ciencias Sociales (Clacso) y la Unesco para construir un sistema de comunicación para las revistas latinoamericanas y del Sur global o la Declaración de Panamá sobre Ciencia Abierta (2018). Se trata de pronunciamientos sintomáticos,

como por ejemplo, RESH (Revistas Españolas de Ciencias Sociales y Humanidades) In-Recs (Índice de Impacto de Revistas Españolas de Ciencias Sociales) o DICE (Difusión y Calidad Editorial de las Revistas Españolas de Humanidades y Ciencias Sociales y Jurídicas). 
no secundados mayoritariamente, que raramente cuestionan el régimen de verdad managerial que impregna la organización, la actividad y los valores de la universidad-empresa actual.

Por otra parte, la dimensión tecnológica tiene también una clara resonancia en el fenómeno de comunicación/evaluación científica. Desde hace unas décadas, el desarrollo de la capacidad técnica -la generalización de repositorios institucionales, la extensión de protocolos de publicación, como OAI (Iniciativa de Acceso Abierto no, necesariamente, gratuito-), la extensión de sistemas de edición de publicaciones (como el Open Journal Systems), etc.- se propone e interpreta como una salida a todo el entramado que captura la producción de conocimiento en universidades e institutos de investigación fortaleciendo el negocio de oligopolios o, directamente, privatizando saberes en cuya base se encuentra el esfuerzo colectivo o la financiación pública.

La tecnología electrónica y de redes que facilita la publicación en línea (por ejemplo, el Directory of Open Access Journals), pareció en algún momento que iba a socavar el poder de los grandes monopolios. Sin embargo, hay que recordar que las políticas de investigación, en el marco europeo al menos, son ambiguas: promueven la publicación en repositorios institucionales o en revistas en libre acceso ${ }^{14}$, pero, de facto, los referentes que siguen contando para catapultar el currículo pasan por los oligopolios citados. Por otra parte, estas corporaciones se adaptaron al medio digital incorporando el open access a sus modelos de negocio: de tal forma que posibilitan publicar en "libre acceso" pero responsabilizan a sus autores y autoras del pago de entre 1.000 y 3.000 dólares/paper. En realidad, es habitual que el sufragio se cargue a presupuestos de proyectos de investigación subvencionados con dinero público. Un proceso que acrecienta las diferencias entre investigadores del Norte-Sur global. Es más, la industria de la comunicación científica ha concentrado las publicaciones en la era digital (Larivière, Haustein, Mongeon, 2015) manteniendo intacta su credibilidad para acreditar el capital simbólico de los investigadores. Por otra parte, aquella cuenta con un potente aliado en la propiedad intelectual, como la Digital Millennium Copyright Act, made in USA, 1998, que impide "colgar" artículos publicados por revistas mainstream.

A nuestro entender, esa perspectiva, aun no careciendo de interés, tiende a reducir la producción y circulación del conocimiento, la trama de saber/ poder, a

${ }^{14}$ Como ocurre, por ejemplo, con el Plan S, 2018, del ERC (European Research Council), apoyado por agencias de financiación y fundaciones privadas (como la Bill and Melinda Gates). 
mera cuestión técnica: pierde de vista la complejidad de las interrelaciones de ese campo de fuerzas -algunas de las cuales se han expuesto- que dificultan que el ideal de "saber universal" en libre acceso y gratuito para el conjunto social, que técnicamente parece posible, ${ }^{15}$ se haga realidad.

El dispositivo de la evaluación funciona como un potente mecanismo de despolitización y (neo)colonización al extender la lógica eficiente y normalizadora sobre países, instituciones o situaciones muy diversos de forma que justifica iniquidades o invisibiliza disfunciones estructurales que deberían evidenciarse, cuestionarse y abordase a través de análisis y mecanismos de naturaleza política. En este escenario el ethos emprendedor del investigador consolida el dispositivo al conformar subjetividades competitivas que dificultan la acción política para enfrentar el dispositivo. Quizá todavía estemos a tiempo para que colectivos y sujetos de conocimiento, no ligados al saber-poder del capitalismo patriarcal y colonial del Norte global, puedan trabajar para suturar las heridas de los epistemicidios y promover un concepto de conocimiento al servicio de "proyectos de vida".

\section{REFERENCIAS}

Amigot Leache, P. y Martínez Sordoni, L. (2013). Gubernamentalidad neoliberal, subjetividad y transformación de la universidad. La evaluación del profesorado como técnica de normalización. Athenea Digital. Revista de pensamiento e investigación social, 13(1), 99-120.

Balanyá, B., Doherty, A., Hoedeman, O., Máanit, A., \& Wesselius, E. (2005). Europe Inc.: comment les multinationales construisent l'Europe et l'économie mondiale. Marseille: Agone.

Ball, S. J. (2012) Performativity, Commodification and Commitment: An I-Spy Guide to the Neoliberal University. British Journal of Educational Studies, 60(1), 1728.

Barsky, O. (2014). La evaluación de la calidad académica en debate: los rankings internacionales de las universidades y el rol de las revistas científicas. Buenos Aires: Teseo.

Beigel, M. F. (2008). David y Goliath, el sistema académico mundial y las perspectivas del conocimiento producido en la periferia. Inchusa-Conicet.

\footnotetext{
${ }^{15}$ En este contexto, desde hace años, se han ido desarrollando proyectos que implican el libre acceso a la documentación, como, arxiv.org, en 1991, la Public Library o Science en 2000 o Sci Hub, fundado por A. Elbakyan, en 2011.
} 
M. Engracia Martín, Comunicación y evaluación del conocimiento...

Facultad de Ciencias Políticas y Sociales. Universidad Nacional de Cuyo. Mendoza.

Bermejo Barrera, J. C. (2006). La inconsistencia de las evaluaciones científicas: elogio del silencio. Revista internacional de filosofía política, 27, 169-186.

Bourdieu, P. y Wacquant, L. (2012). Sobre las astucias de la razón imperialista. En Bourdieu, P., Intelectuales, política y poder (pp. 215-235). Buenos Aires: Eudeba.

Bruno, I. (2008). La recherche scientifique au crible du benchmarking. Petite histoire d'une technologie de gouvernement. Revue d'histoire moderne et contemporaine, 55(4bis), 28-45.

Buranyi, S. (2017, Junio, 27). Is the staggeringly profitable business of scientific publishing bad for science? The Guardian. Recuperado de http://bit.do/e7Uh2.

Callon, M., Courtial, J. P. y Penan, H. (1995). Cienciometría, la medición de la actividad científica: de la bibliometría a la vigilancia tecnológica. Gijón: Trea.

Colectivo Indocentia (2016, Febrero, 19). Disciplinar la investigación devaluar la docencia. Cuando la universidad se vuelve empresa. Entrevista de Fernández-Savater, A. eldiario.es.

Coriat, B. y Orsi, F. (2005, Septiembre, 4). Derechos de propiedad intelectual, mercados financieros e innovación. Sin Permiso.

Craig, R., Amernic, J., \& Tourish, D. (2014), Perverse Audit Culture and Accountability of the Modern Public University. Financial Accountability \& Management, 30, 1-24.

Deleuze, G. (1999). Post-scriptum sobre las sociedades de control. En Conversaciones, (pp. 277-286). Valencia: Pre-Textos.

ERT (European Round Table of Industrialists). (2009). ERT's vision for a competitive Europe in 2025. With recommendations for policy actions. Brusels: ERT.

Estévez Araújo, J. A. (2016). La privatización de la regulación. Papeles de relaciones ecosociales y cambio global, 135, 65-75.

Fyfe, A., Coate, K., Curry, S., Lawson, S., Moxham, N., \& Mork Rostvik, C. (2017). Untangling academic publishing: A history of the relationship between commercial interests, academic prestige and the circulation of research. Zenodo. DOI: http://doi.org/10.5281/zenodo.546100

Gibbons, M., Limoges, C., Nowotny, H., Schwartzman, S., Scott, P., \& Trow, M. (1997). La nueva producción del conocimiento. La dinámica de la ciencia y la 
investigación en las sociedades contemporáneas. Barcelona: Pomares.

Giménez Toledo, E. (2014). Imposturas en el ecosistema de la publicación científica. Revista de Investigación Educativa, 32(1), 13-23.

Gómez Morales, Y. J. (2017). El baile de los que sobran: cambio cultural y evaluación académica. Revista Colombiana de Antropología, 53(2), 15-25.

Gómez Sánchez, L., Jódar Rico, F. y Bravo Sánchez, M. J. (2015). Gubernamentalidad neoliberal y producción de conocimiento en la universidad: genealogía de una configuración subjetiva. Universitas Psychologica, 14(5), 1735-1749.

Hernández Zubizarreta, J. H. y Ramiro, P. (2015). Contra la lex mercatoria, propuestas y alternativas para desmantelar el poder de empresas transnacionales. Bilbao: Icaria.

Herrán, A. de la (2012). Algunas críticas a la evaluación del profesorado universitario centrada en el impacto. En A. de la Herran y J. Paredes (coord.), Promover el cambio pedagógico en la universidad (pp. 305-336). Madrid: Pirámide.

Jessop, R. (2008). El futuro del Estado capitalista. Madrid: Libros de la Catarata.

Kaupinen, I. (2014). The European Round Table of Industrialists and the restructuring of European higher education. Globalisation, Societies and Education, 12(4), 498-519.

Khor, M. (2003). El saqueo del conocimiento, propiedad intelectual, biodiversidad, tecnología y desarrollo sostenible. Barcelona: Icaria.

Larivière, V., Haustein, S., \& Mongeon, P. (2015). The Oligopoly of Academic Publishers in the Digital Era. PLoS ONE, 10(6), e0127502. DOI: https://doi.org/10.1371/journal.pone.0127502.

Mignolo, W. de (2003). Historias locales, diseños globales. Madrid: Akal.

Phillipson, R. (2000). Linguistic imperialism. Oxford: Oxford University Press.

Quijano, A. (1999). Colonialidad del poder, cultura y conocimiento en América Latina. Dispositio. Crítica Cultural en Latinoamérica, paradigmas globales y enunciaciones locales, 23(51), 137-148.

Reig García R. (2014). La investigación dependiente: crítica estructural al sistema JCR. Ámbitos. Revista Internacional de Comunicación, 27, 1-33.

Revista de educación. (1998). La Evaluación de la universidad española. Monográfico, 315. 
Sádaba, I. (2008). Propiedad intelectual. ¿Bienes públicos o mercancías privadas? Madrid: Libros de la Catarata.

Said, E. (1996). Cultura e imperialismo. Barcelona: Anagrama.

Sassen, S. (2007). Una sociología de la globalización. Buenos Aires: Katz.

Shiva, V. (2008). Los monocultivos de la mente. México: Fineo.

Shiva, V. (2003). ¿Progeger o expoliar? Los derechos de propiedad intelectual. Barcelona: Intermón.

Shore, C. (2008). Audit culture and Illiberal governance: Universities and the politics of accountability. Anthropological Theory, 8(3), 278-298.

Sousa Santos, B. de (2010). Descolonizar el saber, reinventar el poder. Montevideo: Trilce.

Unidigna (S.f). Unidigna. Recuperado de http://unidigna.org/

Valdecantos, A. (2009). Reforma universitaria y libertad intelectual. Avances en Supervisión Educativa, 10. 
